Proceedings of the 35th European Peptide Symposium

Patrick B. Timmons, Chandralal M. Hewage, Michal Lebl (Editors)

\title{
Peptides specifically target bacteriophage MS2, filled with an apoptosis-inducing agent, to tumour
} \author{
Igor Sivov 4
${ }^{1}$ Institute of Biomedical Chemistry, Russia
${ }^{2}$ N.F. Gamaleya National Research Centre of Epidemiology and Microbiology, Russia
${ }^{3}$ Russian Research Centre of Oncology, Russia \\ "Biotechnologia" Ltd., Russia
}

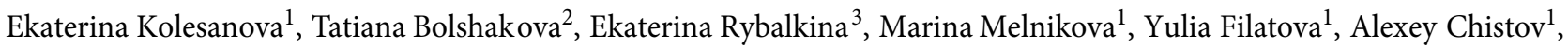

https://doi.org/10.17952/35EPS.2018.300

\section{Summary}

We employed bacteriophage MS2 for the targeted delivery of an apoptosis-inducing agent into tumour tissue. The targeted delivery was provided by iRGD peptides, ligands of integrins presumably located on the surface of tumour tissue novel vasculature endotheliocytes. Peptides were synthesized and conjugated to MS2 capsid proteins. $\mathrm{Tl}^{+}$ions as $\mathrm{TlNO} 3$ were used as apoptosis-induced agents, which penetrated into phage particles and tightly bond to the phage RNA. Peptide-modified MS2 preparations filled with $\mathrm{Tl}^{+}$ caused cell death in several tested solid tumour cell cultures and tumour mass loss in xenograft mice with inoculated with MCF7 and DA-MB-231 cells, while no effect was observed with control preparations(MS2 + peptide, $\mathrm{MS} 2+\mathrm{Tl}^{+}$). Overall concentration of TlNO3 in peptide-modified MS2 filled with $\mathrm{Tl}^{+}$was 500000 times lower than LD50, and therapeutic index of the phage-based preparation was about 15000 . The results show the perspective of the use of bacteriophage MS2 filled with $\mathrm{Tl}^{+}$and targeted by iRGD peptides as a lead substance for solid tumour therapy.

\section{Introduction}

A target-delivering drug system (fig. 1) composed of: 1) a ligand - iRGD peptide (fig. 2), known to bind to $\alpha \mathrm{V} \beta 3$ and $\alpha \mathrm{V} \beta 5$ integrins that are expressed on endothelial cells of the newly formed tumour vasculature and certain tumour cells, and to activate an endocytic/exocytic transport pathway via the interaction with neuropilin-1 [1]; peptide ligands contained Gly3 spacers for their conjugation with phage particles; 2) a vehicle - bacteriophage MS2 with a single-stranded genomic RNA, replicating only in F-pilus-bearing enteric bacteria that are neither symbiotic nor pathogenic for human organisms. MS2 capsid contains pores permeable for metal ions $\left(\mathrm{Tl}^{+}\right)$. MS2 serves an impermeable vehicle for $\mathrm{Tl}^{+}$, excluding its toxic action on tissues except tumour and its vasculature; 3 ) an active substance $-\mathrm{Tl}^{+}$, a toxic metal ion known to cause cell apoptosis. $\mathrm{Tl}^{+}$ tightly binds to viral RNA and hence does not leak from MS2 particles [2]. It is not removed from cancer cells via multidrug resistance-responsible systems [3].

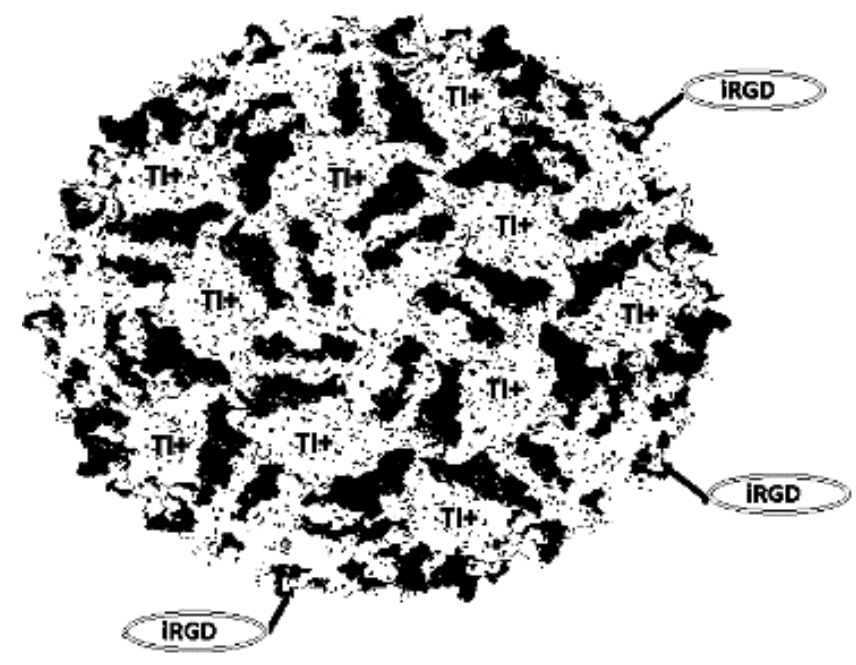

Figure 1: A schematic image of bacteriophage MS2 conjugated with iRGD peptide and filled with Tl+. 


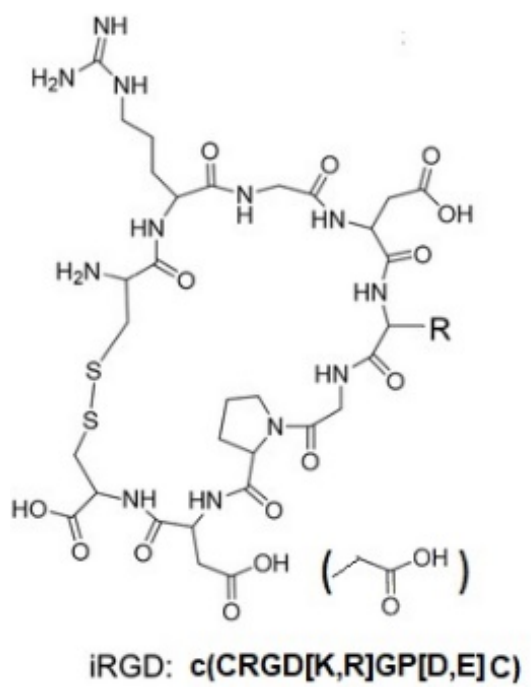

Figure 2: Structure of iRGD peptides

\section{Experimental}

iRGD peptides were prepared by automated SPPS (433A peptide synthesizer, Applied Biosystems) on 2-ClTrityl resin from Fmoc-amino acids via FastMoc procedure (activators - HBTU with Oxyma Pure); cysteine derivatives (Trt- and Acm-protected) were coupled with DIC/Oxyma Pure. Cyclization via formation of S$\mathrm{S}$ bridges was achieved on totally protected peptides in solution by $\mathrm{I}_{2}$. After the cyclization the peptides were deprotected, purified by reverse-phase HPLC and characterized by LC-MS for the conformity of their structure and purity. Peptides were conjugated to MS2 phage particles by using a bifunctional reagent dimethyl adipimidate through protein and peptide free $\mathrm{NH}_{2}$ groups [4]. Filling of peptide-MS2 particles with $\mathrm{Tl}^{+}$was achieved by incubating peptide-conjugated MS2 $\left(3.3 \times 10^{10} \mathrm{pfu}\right.$ (plaque forming units)/mL) with $0.5 \mathrm{mkM} \mathrm{TlNO}_{3}$ with further precipitation by polyethylene glycol 6000 and dialysis. $\mathrm{Tl}^{+}$dosage was $5 \times 10^{-7}$ g-equiv per 1 pfu (determined fluorimetrically and mass-spectrometrically). Four different preparations of peptide-conjugated MS2 filled with $\mathrm{Tl}^{+}$containing four different iRGD peptides were mixed in about equimolar ratios for further testing. Testing of the drug delivery system was performed on cultivated MCF-7 (hormone-dependentbreast cancer) and DA-MB-231 (hormone-independent breast cancer) cells and nude mice with inoculated MCF-7 and DA-MB-231 xenografts. Cell viability was tested in serum-free (enhanced phagocytosis) and serumcontaining media by Evans Blue staining. Effect on animal xenografts was evaluated after 10 p.o. or i.p. injections of $10^{8} \mathrm{pfu} / \mathrm{kg} /$ day by comparing tumour area in experimental and control animal groups. PeptideMS2 without $\mathrm{Tl}^{+}$and MS2 $\mathrm{Tl}^{+}$without peptides were used as controls.

\section{Results and Discussion}

iRGD peptides were prepared in $95 \%$ purity. Lys $^{8}$-containing iRDG peptides formed intermolecular rather than intramolecular S-S bridges by air oxidation of deprotected peptides and did not readily formed S-S bridges while on resin, and we could successfully cyclize these peptides only in a totally protected state. $\mathrm{Arg}^{8}$-containing peptides were prepared by the same scheme as well. Incubation of peptide-conjugated MS2 particles in $\mathrm{TINO}_{3}$ solution at neutral $\mathrm{pH}$ resulted in the drop in $\mathrm{Tl}^{+}$concentration in the media up to 30 times compared to the initial one, thus representing the filling of the phage paricles with $\mathrm{Tl}^{+}$, which was tightly bound to the phage RNA. Free $\mathrm{Tl}^{+}$content of the preparationwas $<10 \%$, while $\mathrm{Tl}^{+}$content inside phage particles was about 5x10-11 g-equiv./pfu. An equivalent of $10^{8}$ pfu peptide-modified MS2 filled with $\mathrm{Tl}^{+}$added to cell cultures resulted in $80-85 \%$ both MCF7 and DA-MB-231 cell death in enhanced phagocytosis conditions, with much lower apoptosis in serum-containin gmedia. No cell death was observed in control experiments. When peptide-modifiedMS2 filled with $\mathrm{Tl}^{+}$was injected to MCF7- and DA-MB-231-x enograft nude mice, it caused tumour mass loss up to 2.5 times compared to control (fig. 3). 


\section{Before injections}

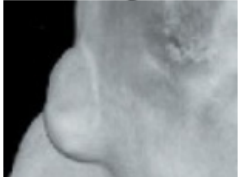

\section{After injections}
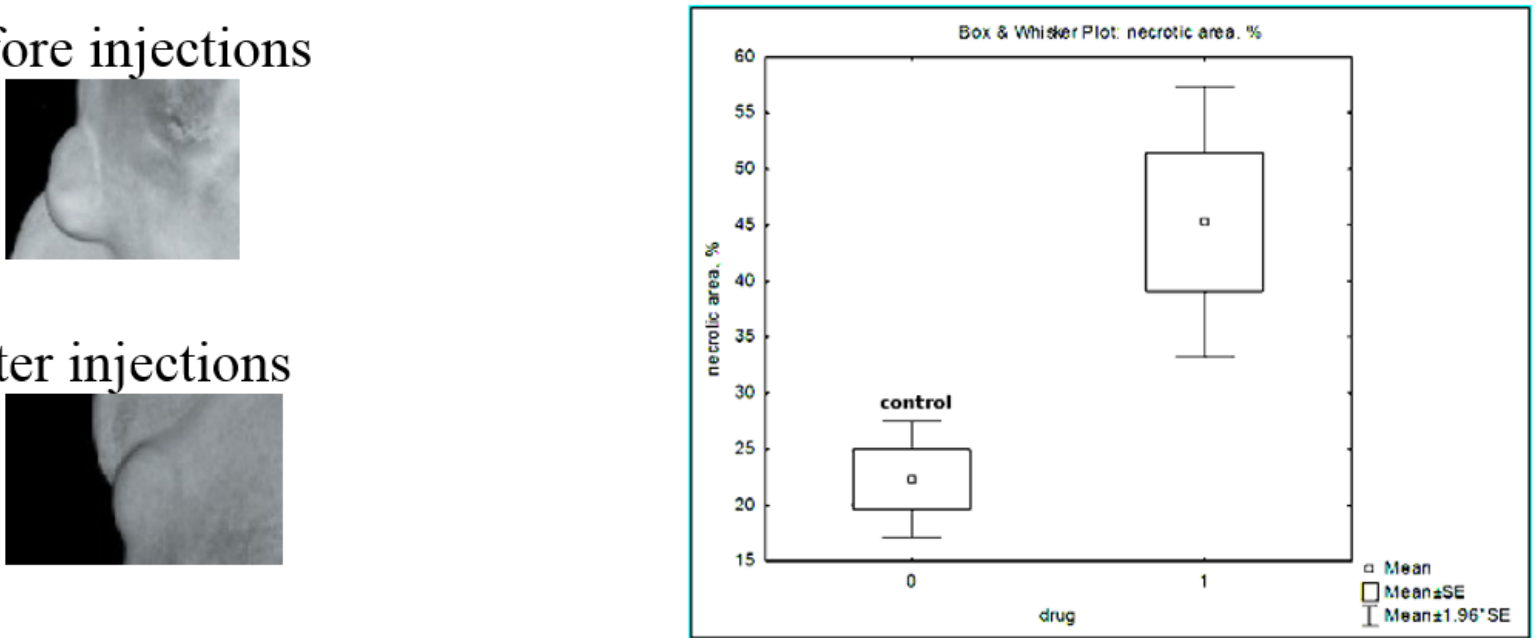

Figure 3: Tumour necrosis areas visualized on photographs and determined by ScanScope CS2

$\mathrm{LD}_{50}$ for iRGD-MS2-Tl was estimated as $1.5 \times 10^{8} \mathrm{pfu} / \mathrm{kg}$ in Wistar-Kyoto rats. Overall concentration of $\mathrm{TlNO}_{3}$ in peptide-modifiedMS2 filled with $\mathrm{Tl}^{+}$was 500000 times lower than $\mathrm{TlNO}_{3} \mathrm{LD}_{50}$, and therapeutic index of the phage-based preparation was about 15000. The results show the perspective of the use of bacteriophage MS2 filled with $\mathrm{Tl}^{+}$and containing a target delivery system as a lead substance for solid tumour therapy. $\mathrm{No} \mathrm{Tl}^{+}$leakage was observed during iRGD-MS2-Tl storage. Hence iRGD-MS2-Tl was recommended for preclinical investigations as an anticancer drug for the treatment of breast cancer.

\section{Acknowledgments}

The research was supported by the State contract with the Russian Ministry of Education and Science No. 14.N08.11.0188 (27.11.2017) (unique project ID 171771053913577100100100030807219241). Peptides were synthesized with the use of the equipment of "Human Proteome" Core Facility of the Institute of Biomedical Chemistry (Moscow, Russia) supported by Ministry of Education and Science of the Russian Federation (unique project ID RMEFI62117X0017). Animal experiments were performed at the N.N. Petrov Research Oncology Centre of the Russian Ministry of Healthcare.

The results are covered by the Russian Federation Patent No. 2599462, PCT WO 2017052419, and US patent claim $15 / 757,285$ of 02.03 .2018 .

\section{References}

1. Tessalu T. et al. / Proc. Natl. Acad. Sci. USA 106 (2009) 16157-16162. doi: 10.1073/pnas.0908201106.

2. Zasukhina G.D. et al. /Mutat. Res. 124 (1983) 163-173

3. Korotkov S. M., et al. /J. Biochem. Mol. Toxicol. 28 (2014) pp. 149-156. doi: 10.1002/jbt.21547.

4. van Regenmortel M.H.V., Muller S. Synthetic peptides as antigens. Elsevier, 1999, pp. 88-90. 